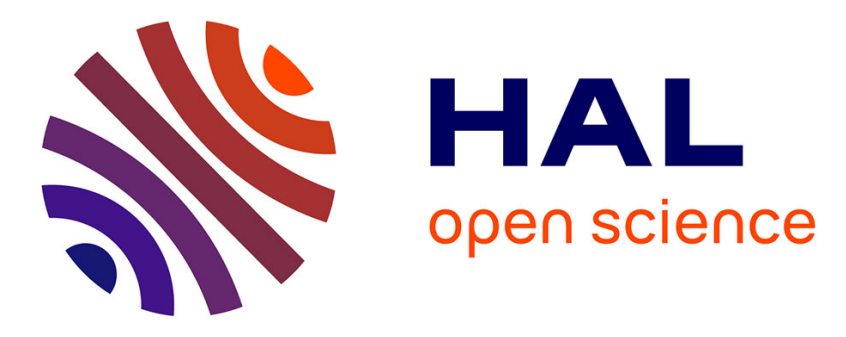

\title{
Remote Control of an Assistive Robot using Force Feedback
}

\author{
Paul Nadrag, Lounis Temzi, Hichem Arioui, Philippe Hoppenot
}

\section{To cite this version:}

Paul Nadrag, Lounis Temzi, Hichem Arioui, Philippe Hoppenot. Remote Control of an Assistive Robot using Force Feedback. 15th International Conference on Advanced Robotics (ICAR 2011), Jun 2011, Tallin, Estonia. pp.211-216, 10.1109/ICAR.2011.6088570 . hal-00594851

\section{HAL Id: hal-00594851 \\ https://hal.science/hal-00594851}

Submitted on 21 May 2011

HAL is a multi-disciplinary open access archive for the deposit and dissemination of scientific research documents, whether they are published or not. The documents may come from teaching and research institutions in France or abroad, or from public or private research centers.
L'archive ouverte pluridisciplinaire HAL, est destinée au dépôt et à la diffusion de documents scientifiques de niveau recherche, publiés ou non, émanant des établissements d'enseignement et de recherche français ou étrangers, des laboratoires publics ou privés. 


\title{
Remote Control of an Assistive Robot using force feedback
}

\author{
Paul Nadrag, Lounis Temzi, Hichem Arioui, Philippe Hoppenot \\ paul.nadrag@ibisc.univ-evry.fr \\ IBISC, Evry University, FRANCE
}

\begin{abstract}
In this paper, we consider the haptic teleoperation of an assistive mobile robot, used for exploring a domestic environment. The goal of the paper is to help the remote operator to pilot the robot by giving him not only video feedback but also haptic feedback. They are both complementary as they do not require the same kind of attention from the user. The proposed haptic architecture was found to improve operator perception of the remote environment under time delay communication. The humanoperator can control actively the mobile robot, using its intrinsic sensors, and "feel" the slave environment. Experimental results, with a real robot, are performed and analyzed.
\end{abstract}

Keywords: Multimodal interface, force feedback teleoperation, assistive robotics, mobile robot remote control, transmission delays.

\section{INTRODUCTION}

The increasing number of elderly people, especially with pathologies such as Alzheimer disease, is becoming an important issue in Europe. It is more and more difficult and expensive to assure long term hospitalization for these people, so they stay at home as long as possible. There are two main issues that must be solved in order to make that possible: the security of the person and cognitive stimulation. The aim of the European CompanionAble project (www.companionable.net) is to assist the people with Mild Cognitive Impairment (MCI) and their families in those situations, in the context of ambient assisted living. In this context, caregivers and relatives can pilot the robot though the Internet, allowing them the possibility to have distant interaction with the user. The purpose of the robot is not to remove the human presence around the person, but to ease his caring. Decreasing this presence is a clearly expressed wish, in a quite comprehensible will of intimacy for the person and in a will of assistance for the family.

A multimodal interface is a good way to give to the remote operator as many kinds of information as possible to perceive the distant scene, where the robot evolves. With the spread of low-cost haptic devices, haptic interfaces have been used in many areas of robotics and recently in the field of mobile robot teleoperation ([1], [2], [3]). These mobile robots operate in unknown and dangerous environments performing particular tasks. Haptic devices help to improve the operator perception of the environment and give users the illusion of "feeling" the robot workspace, improving, among others, his/hers obstacle avoidance performance and reducing the number of collisions [3].

These applications find all their interest in slave remote environments, namely the human intervention in one or more remote sites. But this distance induces the major problem of stability and transparency, due to communication delay. In this paper we focus on haptic feedback. We especially study, in part, the impact of the transmission delay and we adapt a predictive control scheme [4] to overcome instability problems.

This paper is organized as follows: next section is dedicated to works related to our topics. In the following one we present the proposed control scheme. Section 4 presents experimental results performed on a real robot. Last section is for the conclusion and future work.

\section{RELATED WORKS}

The most important problem in remote controlled tasks comes from sensorial impoverishment because of the separation between the entity which controls the action (human being) and the entity which executes it (the machine). Figure 2.1 illustrates a remote control situation in which the human operator is far away from the area where the action takes place. The most common solution to solve the problem is to elaborate a humanmachine co-operation. Both human being and machine have the abilities of perception, decision and action. They can help each other to realize the mission desired by the operator, by the mean of shared control modes of the robot ([5], [6]). In normal situation, humans exploit a great diversity of sensorial information (visual, aural, tactile, vestibular, etc.). In remote control situation, some of them are degraded or totally absent. Two of them are overexploited: vision and proprioception. In this paper we deal with proprioception, which deals with low cognitive level feedback to the user.

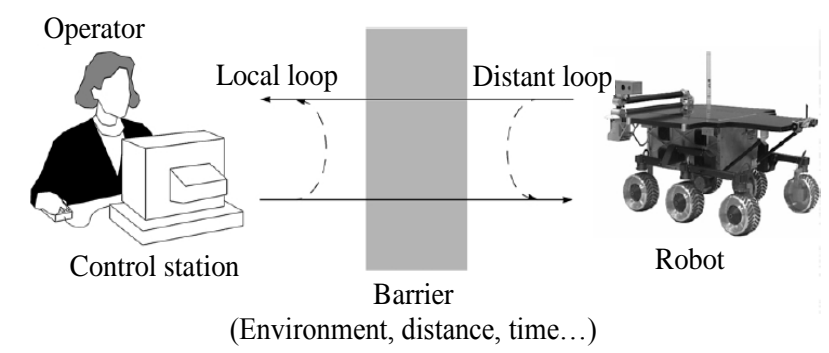

Figure 2.1. Remote control situation (adapted from Fong et al. (2001)

[7]).

Our application consists of a teleoperated mobile robot navigating in a real environment. This implementation is similar to a teleoperation system with the only difference of the used interface (master robot): Phantom Omni. This sole difference does not make our application a haptic interaction, unless if we interact with a virtual environment, which can be a representation of the real scene, actually provided. For this reason, we discuss in the rest of the paper of haptic interaction and not teleoperation.

Different papers have been done on mobile robot teleoperation with force feedback. In [8], forces are used in the feedback to the user but also for obstacle avoidance. Authors had 
experimented their proposition in a virtual environment, comparing three conditions: no force feedback, force feedback without obstacle avoidance and force feedback with obstacle avoidance. They have shown that haptic information improves operator performance in terms of number of collisions and distance to obstacles without degeneration for navigation time. Authors of [2] propose another way to compute the force, taking into account not only an elastic repulsive force but also a viscous friction. This has been implemented not only in simulation but also on a real robot. In [1], authors have unveiled a theoretical technique of how to stabilize such an application in the presence of a constant delay without real validation. In [9] and [10], authors proposed to take into account not only the distances between the robot and its environment but also the speed of the robot. That improves the quality of motion control. In all these cases, transmission delays between the master site and the slave site have been neglected. In [11], authors address the issue of transmission delays between the master site and the slave site. They propose a Self-Organizing Fuzzy Adaptive Mapping algorithm. They have implemented it on a real robot with small transmission delays (less than 0.15 seconds).

The bilateral control schemes used for conventional haptic interactions are inherited from the teleoperation. Several studies have been conducted on these aspects of bilateral control algorithms. It should be noted that these control schemes are valid but not sufficient if communication delay exists. To solve the stability problems that occur in this case, researchers have added other layers of command laws. A first team [12] introduces a decentralized controller that can further enhance the teleoperation transparency. A second team proposes a wave-variables scheme to overcome the stability problem of interconnected passive systems ([13], [14]) under communication delay. Other researches use techniques based on predictive control ([15], [4]) to enhance the failures and performance degradations in terms of position tracking.

\section{MODEL OF CONTROL SCHEME}

First works in haptic for robots dealt with manipulator arms. Based on previous findings in bilateral control of teleoperators, the "Rate-Force" control scheme is the most suitable for our application. This is motivated by, among other, the kind of haptic device used. Thus, the communication protocol of data transfer (input / output) is imposed by the robot Lina (described later in the paper).

Moreover, an amendment was made to the control model by using the haptic interface position as a set point speed (linear and angular) to the remote mobile robot. This change allows the interface to emulate a joystick. In this manner, the haptic device receives the force feedback calculated from ultrasonic data.

\subsection{Impedance control scheme}

In this section, we explain how the data transfer between all components of our experiment is done.

Figure 3.1 highlights the functioning of our system. The human operator $F_{h}$ is manipulating the haptic end-point to move the remote mobile robot. The performed motion $X_{m}$ will be translated by a set of linear velocity $V_{r}$ to the mobile robot. The performed motion $Y_{m}$ will be translated by a set of angular velocity $\Omega_{r}$ to the mobile robot. The homothetic scheme (equation below) is applied between different variables to find a best match with the velocities of the mobile robot, such as:

$\left\{\begin{array}{l}V_{r}=k_{v} \cdot X_{m}(t-\tau(t)) \\ \Omega_{r}=k_{\Omega} \cdot Y_{m}(t-\tau(t))\end{array}\right.$

Where $V_{r}$ and $\Omega_{r}$ are respectively the linear and the angular velocities of the mobile robot, $k_{v}$ and $k_{\Omega}$ are the homothetic coefficients applied respectively to displacements $X_{m}(t-\tau(t))$ and $Y_{m}(t-\tau(t)) . \tau(t)$ is the variable time delay.

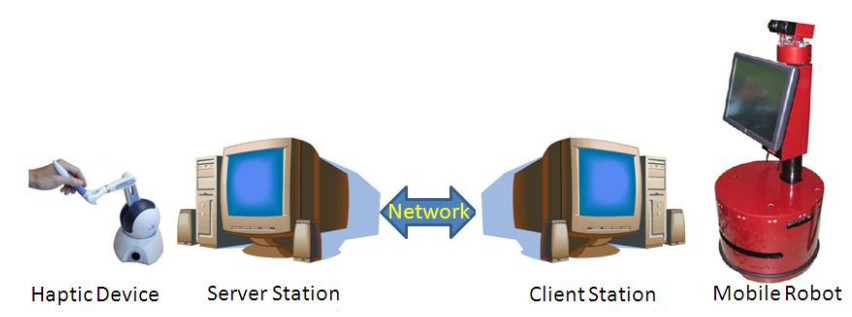

Figure 3.1. Simplistic scheme of the haptic interaction

\subsection{Force Feedback Strategy}

As stated before, the force feedback computing is based on the $X i$ distance, between the mobile robot and obstacles, measured by 7 ultrasonic sensors instrumented on the front of the mobile robot (Figure 3.2). The phase shift between each couple of sensors is $30^{\circ}$.

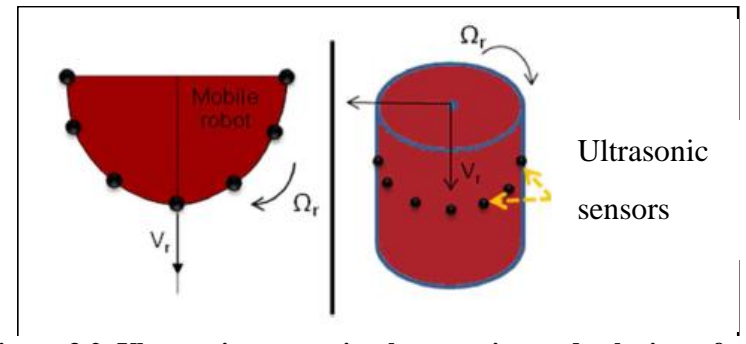

Figure 3.2. Ultrasonic sensors implementation and velocity references In order to compute the necessary force to alert the human operator of the presence of an obstacle or an impending impact, we set two threshold distances from which the operator feels two different forces (Figure 3.4). These levels are defined as follows:

$\left\{\begin{array}{c}X_{\text {wall }} \leq X_{i} \leq X_{\text {spring }} \Rightarrow F_{i}=k_{s}\left(X_{i}-X_{\text {spring }}\right) \\ 0 \leq X_{i} \leq X_{\text {wall }} \Rightarrow F_{i}=k_{w}\left(X_{i}-X_{\text {wall }}\right)+k_{s}\left(X_{\text {spring }}-X_{\text {wall }}\right)\end{array}\right.$

Where $X_{\text {spring }}$ is the first threshold position limiting the "spring" zone, $X_{\text {wall }}$ is the first threshold position limiting the "wall" zone, $k_{w}$ and $k_{s}$ are stiffness coefficients calculated depending on the zone properties.

The overall forces $F_{r}$ delivered to the operator are given by:

$F_{r}=\sum_{i=0}^{6} F_{i}$

By projection onto the $X$ and $Y$ axes, we get: 


$$
\left\{\begin{array}{c}
F_{r / x}=F_{1} \sin \left(\frac{\pi}{6}\right)+F_{2} \sin \left(\frac{\pi}{3}\right)+F_{3}-F_{4} \sin \left(\frac{\pi}{3}\right)-F_{5} \sin \left(\frac{\pi}{6}\right) \\
F_{r / y}=F_{0}+F_{1} \cos \left(\frac{\pi}{6}\right)+F_{2} \cos \left(\frac{\pi}{3}\right)-F_{4} \cos \left(\frac{\pi}{3}\right)-F_{5} \cos \left(\frac{\pi}{6}\right)-F_{6}
\end{array}\right.
$$

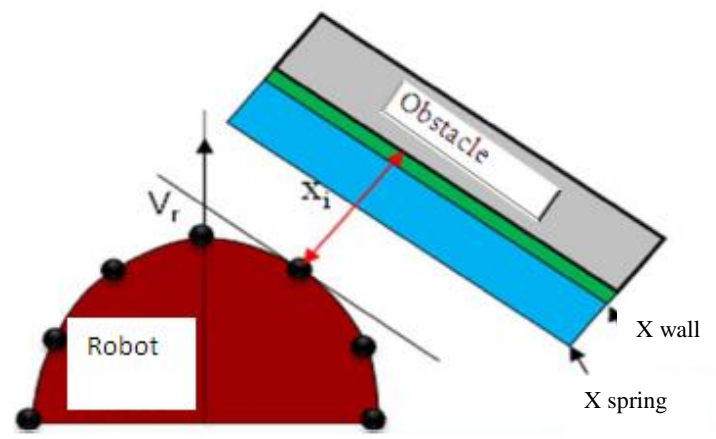

Figure 3.3. Threshold distance limit between mobile robot and obstacle.

\subsection{Joystick effect}

For security reasons and to safeguard the mobile robot, we have added a permanent force feedback $\mathrm{F} 0 /(\mathrm{x}, \mathrm{y})$ so that it repositions the haptic end-point in its neutral position or neutral zone. This property is important if the operator releases the haptic arm, the end-point quickly returns to the neutral position and the robot stops its progression.

$$
F_{0 /(x, y)}=-k_{0} X_{m}
$$

Where: $k_{0}$ is a small stiffness.

The neutral position can be defined as a neutral zone to preserve the life of the engines against hand tremors.

\subsection{Delays compensation}

This section addresses the stability of the time-delayed haptic interaction with real/virtual environment systems. A stable predictive-like approach is proposed (inspired by the Smith predictor, [15], [18]). Neither time delay estimation nor time delay behavior knowledge is required. The controller can be applied to constant or time-varying delays without any adaptation.

In Figure 3.4, $M(s)$ is the haptic device transfer function, $C(s)$ is the local controller (virtual coupling, [16]), $\tau_{i}$ are respectively upstream and downstream time delays (taken constant on the figure) and $E(s)$ represent the robot and its environment. $F_{e}$ is the slave environment computed force; $F_{h}$ is the operator-applied force on the device.

Figure 3.4 illustrates the evolution of the controller (see [4] for more details). The first step was to apply the principle of the Smith predictor model on the slave environment. This idea was quickly ignored because of the difficulty of predicting the behavior of the robot and its dynamic environment. Therefore, we applied the same principle around the master device (the model of the master device should be linear and well known). To succeed in this latest development, we must know the value of the delay (when it is constant) and even more difficult to predict the change (if it varies). This second case is very interesting, but unusable if we use a non-deterministic communication protocol (such as the Internet) without an appropriate control law.

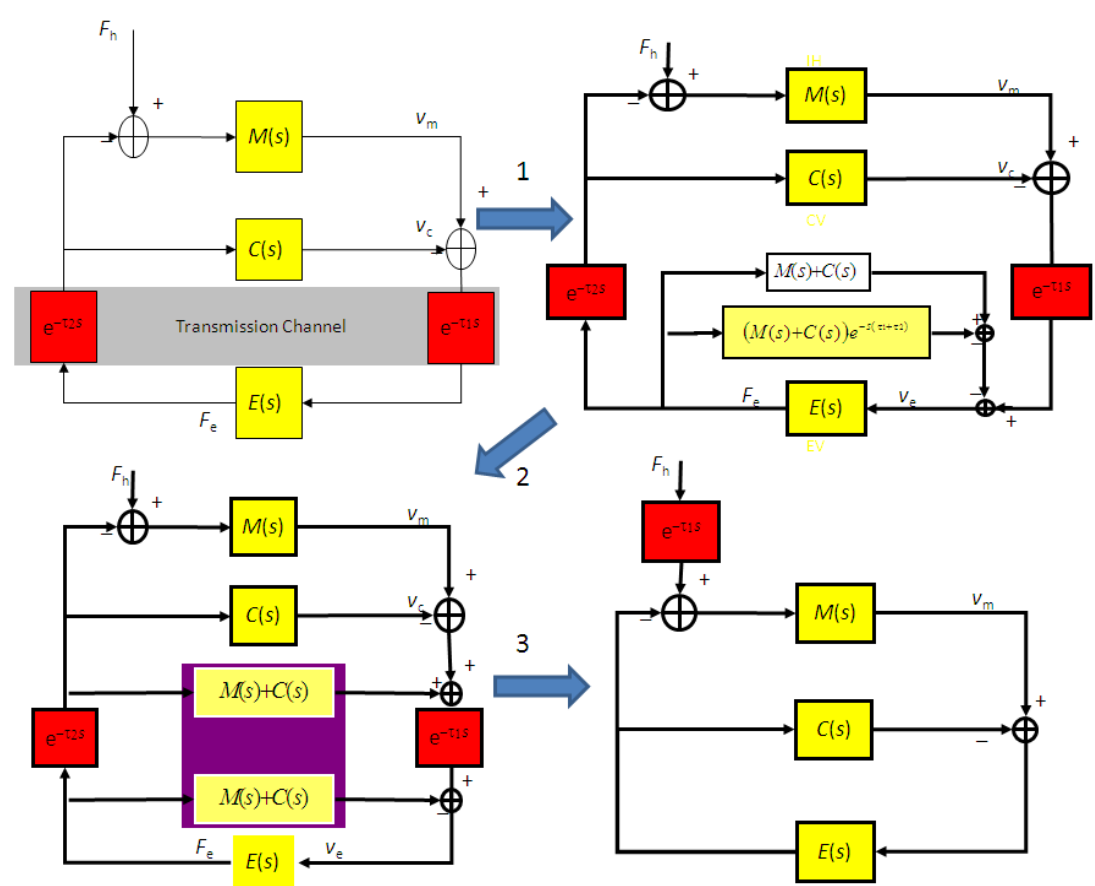

Figure 3.4. Predictive Control design steps: (a) Delayed haptic interaction without control, (b) Smith predictor application, (c) Modified Smith predictor (information on delay size unnecessary) and (d) final equivalent control scheme.

consists of a stable controller requiring only knowledge of the

To overcome this difficulty, we move the second delayed haptic device model. This evolution suggests that stability in the case of variable delay is maintained ([17] for proof). 


\section{EXPERIMENTAL RESULTS}

\subsection{Experimental protocol}

All the experiments have been performed on a real robot. Lina (Figure 4.1) is a circular two driving wheels robot $(55 \mathrm{~cm}$ of diameter). Its maximum speed is $1.2 \mathrm{~ms}^{-1}$ and $4 \mathrm{rds}^{-1}$. Optic coders are used to compute odometry. It is equipped with 12 ultrasonic sensors all around its body, one each $30^{\circ}$. For the present experimentations, only the seven frontal sensors are used. They are labeled $x 0$ to $x 6$, from the left to the right. A laser range finder is also present in the front $\left(210^{\circ}\right.$ of aperture) of the robot. A pan-tilt camera is used for video feedback to the operator. The robot uses a Wi-Fi connection. Speed orders are sent to the robot through this connection. Optional functions such as obstacle avoidance are available through a human-machine interface displayed on the touch screen of the robot.

On the operator side, we use a Phantom to generate force feedback. The video and force feedbacks have the same priority.

We have validated our model in three classical spatial situations encountered in indoor environments: movement towards a wall (Figure 4.2.a), following a corner wall (Figure 4.2.b) and driving between two obstacles (Figure 4.2.c).

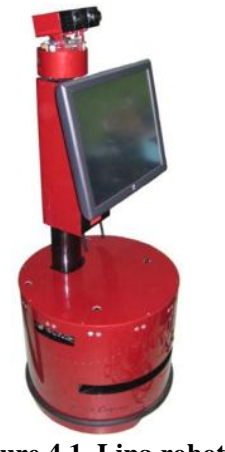

Figure 4.1. Lina robot.

For each of these spatial situations, four kinds of feedbacks to the human operator were tested. In the first one, no force is sent to the user. This situation is called "NF" (No Force). It is a reference experiment, in which forces are computed but not fed back to the operator. That will be a mean to compare this situation with the others. In the second situation, a force is fed back to the operator, without transmission delay. This situation is called "ND" (No Delay). The third situation corresponds to a force sent with a delay. This situation is called "DNC" (Delay Not Corrected). The last situation corresponds to a force sent to the user with a delay, modified by our corrector (Delay Corrected). In the DNC case, the system computes the force feedback in the same way than in the case without delay. In the DC case, the system takes the delay into account.

48 trajectories, considering the 3 spatial situations and the 4 feedback conditions, have been performed by subjects aged between twenty-five and thirty. They were students in the lab, familiar with robots and remote control, but not with force feedback. Subjects had about twenty minutes to familiarize with the force feedback. This learning was conducted without transmission delays. All subjects were asked to pilot the robot in each spatial situation under each feedback condition. In spatial situation 1 (Figure 4.2.a), subjects had to drive up to the wall. In spatial situation 2 (Figure 4.2.b), subjects were asked to follow the first wall corner, turn left and follow the second wall. They then had to drive back to the start point. In the last spatial situation (Figure 4.2.c), subject had to drive the robot between the two obstacles and come back at the starting point.

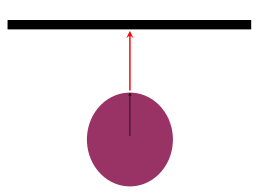

(a)

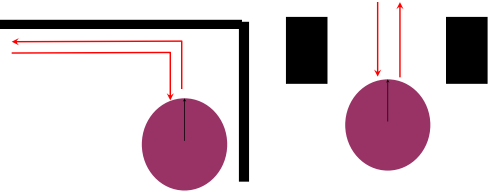

(b)

(c)

Figure 4.2. Classical spatial situations encountered in indoor environment.

\subsection{Results}

We have taken into account tree criteria to analyze these experiments from the operator point of view:

1. The time to realize a mission

2. The force variations

3. The command variations

ANOVA analysis was used to determine if differences between situations, regarding each criterion, was significant. $p$ factor determines the significance. It is generally accepted that if $\mathrm{p}<0.05$, the result is statistically significant.

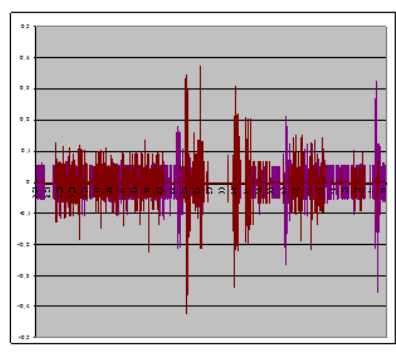

NF

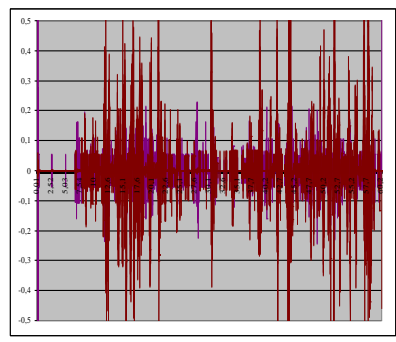

DNC
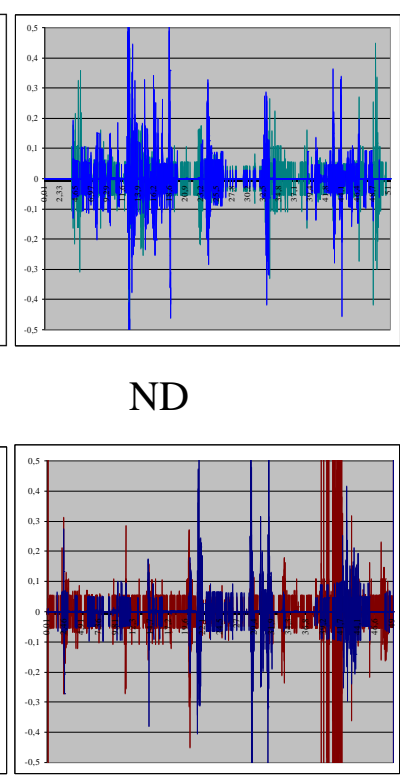

DC

Figure 4.3. Illustration of phantom accelerations in the four tested situations.

As trajectory lengths are different regarding the situations, we have used well identified parts of the trajectories which have the same characteristics. Results on time necessary to realize a mission show that there is no significant difference between $\mathrm{NF}$ and $\mathrm{ND}(\mathrm{p}=0.62), \mathrm{NF}$ and $\mathrm{DC}(\mathrm{p}=0.93)$. The difference is also not significant between NF and DNC but the significance is not so high $(\mathrm{p}=0.08)$. 
We have also analyzed how many times one can observe a threshold (higher than $0.5 \mathrm{~N}$ ) in the variation of the force feedback. That gives information on the perturbation felt by the user. As explain above, even if the force is not returned to the user in the "NF" case, it has been calculated. That gives us a mean to compare the different situations. We obtain the same kind of results: "NF" is not significantly different from "ND" ( $p=0.25)$, "DC" $(p=0.91)$ and slightly not different from "DNC" $(p=0.07)$. More, the conditions with returned effect are not significantly different between each others: "ND" and "DC" (p=0.40), "ND" and "DNC" $(p=0.20)$ and "DC" and "DNC" $(\mathrm{p}=0.09)$.

The third quantitative criterion deals with the variation of the command generated by the operator with the Phantom. We have measured accelerations of the Phantom variations (the instability of the control). We have measured the numbers of acceleration higher than $0.2 \mathrm{~ms}^{-2}$. We have found that $\mathrm{NF}$ (without force feedback) condition is very significantly different from the others (with force feedback): "NF" vs "ND" $(p=0.007)$, "NF" vs "DC" $(p=0.003)$ and "NF" vs "DNC" $(p=0.006)$. If we compare the conditions with force feedback, we find that "ND" vs "DNC" is significantly different $(p=0.02)$ in the way that the command is more jerky with the non corrected delay. "ND" vs "DC" is not significant $(p=0.27)$ and "DC" vs "DNC" is just in the limit $(p=0.054)$. Figure 4.3 shows the accelerations of the Phantom for one spatial situation (described in Figure 4.2.c) with all the proposed feedbacks. One can notice than with "ND" feedback, accelerations are often inferior to $0.2 \mathrm{~ms}^{-1}$. One can also observe than accelerations are very often superior to $0.2 \mathrm{~ms}^{-1}$ in "DNC" case. Finally, accelerations look like similar in "ND" and "DC" cases. That is only an illustration of the results described above.

We also have asked the operator to share their feelings about force feedback. In the first situation (Figure 4.2.a) force feedback is not judged very useful as visual feedback gives good information. In the other situations (Figure 4.2.b and Figure 4.2.c), force feedback seems to be appreciated as it gives information that are not given by video feedback (the obstacles are not in the camera's field of view). In those cases, transmission delays disturb the users if they are not corrected. A drawback is that the feeling of force feedback is not very clear in diagonal directions.

Finally, Figure 4.4 illustrates the efficiency of the proposed predictive control strategy to compensate transmission delays (here $200 \mathrm{~ms}$ up and down). Forces fed back to the operator in $\mathrm{X}$ and $\mathrm{Y}$ axes are represented. Figures show that three situations generate similar force feedback: NF (forces are computed but not fed back to the user), "ND" (forces with transmission delays), and "DC" (forces with transmission delay and predictive control). The fourth situation, "DNC" (forces with transmission delay and without control) induces vibrations which are very disturbing for the operator.
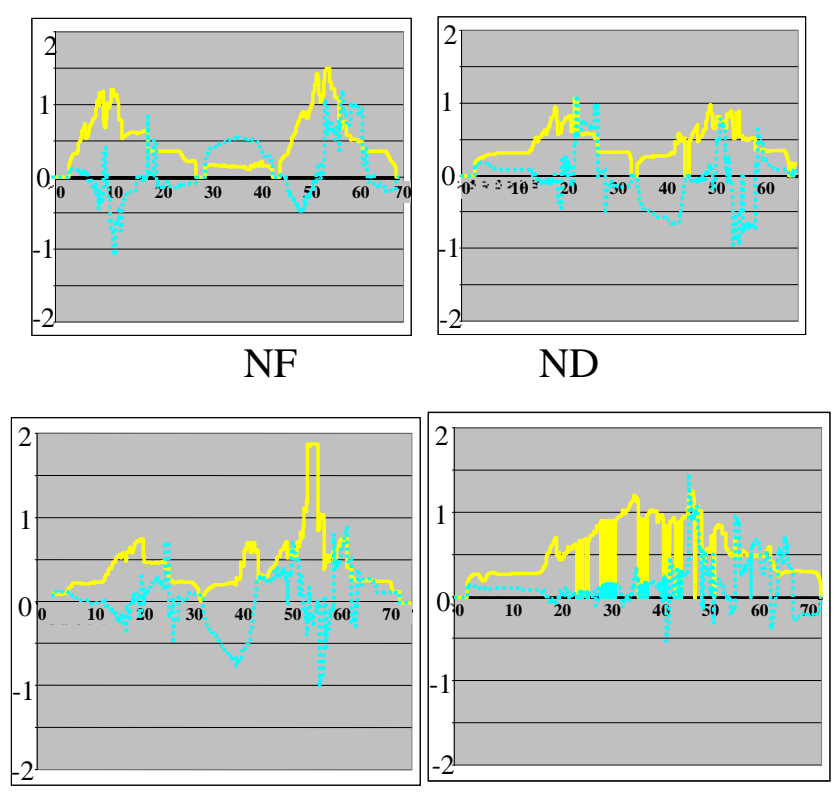

\section{DNC}

DC

Figure 4.4. Experimental result illustrating delays compensation on force feedback. $\mathrm{X}$ axis represents time in seconds and $\mathrm{Y}$ axis represents force in Newton.

\subsection{Discussion}

On the one hand, considering the first two statistical criteria, one can say that it is not very clear if force feedback helps the operator in that configuration of remote control. Indeed, time taken to execute a trajectory is not better with force feedback, even without transmission delays. More, the forces computed in all the situations are not significantly different. That does not give arguments in favor of force feedback for wheeled robot remote control. In fact, the force feedback model is not very natural for the user. Indeed, the commands sent by the operator depend on the position of the Phantom and these commands are interpreted as speeds by the robot. The issue is that when the operator feels a force in the Phantom, if he stops his command movement, the robot is not stopped. That is not the case in with manipulator arms in which the position of the slave arm is directly connected to the position of the master unit.

On the other hand, the third criterion studied above shows a significant difference of behavior of the user, which is in favor of the force feedback assistance. Indeed, that shows that the behavior of the operator is affected by the force feedback, meaning that the information is useful for the user.

The operator feels that force feedback is interesting to deal with information that is not present in the video feedback. But the feeling in the diagonal directions is not very easy to understand. The exact orientation of the obstacles are difficult to appreciate and the fact that the robot keeps on moving if the operator just stops his movement without putting the Phantom in its central position is not very natural to deal with.

\section{CONCLUSION AND FUTURE WORK}

In this paper, an assistive robot teleoperation system with haptic interface has been presented, to enrich the multimodality of the remote control interface, which 
classically used video feedback and distance information given by ultrasonic sensors and laser range finder. The application goal is the remote control of a mobile robot to perform an exploration task in a domestic environment. The proposed control scheme uses the X-Y displacements of the haptic device as a speed control for the mobile robot. For security reasons we have implemented a standard joystick control to preserve the robot's motors from damages.

Our initial objective was to analyse delay consequences on remote control using force feedback and to correct them. We achieved two main points. The first one is the stabilisation of the command. This is shown by the fact that the command generated by the user is the same in ND (force feedback without delay) case and DC (force feedback with corrected delay) case, which are different with DNC case (force feedback with uncorrected delay). This is measured by the fact that accelerations generated by the Phantom are significantly more important in the last case (DNC) than in the two former ones (ND and DC). The second point is that force feedback is described as interesting information by the operators for information that is not given by the video feedback.

During the analysis, we have seen that, according to certain criteria, force feedback (with and without delay) does not seem to have benefits. We think it is interesting to share this type of results. That can be explained by the fact that positions in the master space are transformed in speeds in the slave space. One way to deal with this problem could be to progressively switch from a position-speed control to a position-position control when the robot comes close to an obstacle.

Interviews with the users show that they feel interested to have more information from their environment than just the video feedback. But they also noticed that diagonal information is not very easy to understand through the Phantom. Regarding the stability of the command under delay, the proposed system works perfectly to stabilize the command, which is an important point.

Future work on this topic will be to explore other control possibilities to permit the operator not only to feel the obstacle but also to feel more natural the interaction, regarding the fact that when the operator stops moving, the robot can keep on moving depending on the position of the Phantom, not on its speed.

\section{ACKNOWLEDGMENTS}

The research leading to these results has received funding from the European Community's Seventh Framework Program ([FP7/2007-2013]) under grant agreement no. 216487 (CompanionAble: http://www.companionable.net/).

\section{REFERENCES}

[1] Lee D., Martinez-Palafox D. and Spong M. W., "Bilateral Teleoperation of a Wheeled Mobile Robot over Delayed Communication Network", IEEE Int. Conference on Robotics and Automation, Orlando, Florida, 2006.
[2] Diolaiti N. and Melchiorri C., "Tele-Operation of a Mobile Robot Through Haptic Feedback", IEEE Int. Workshop on Haptic Virtual Environments and Their Applications (Have'02), Ottawa, Ontario, Canada, 2002.

[3] Mitsou, N. C., Velanas S. V. and Tzafestas C. S., "VisuoHaptic Interface for Teleoperation of Mobile Robot Exploration Tasks", $15^{\text {th }}$ IEEE Int. Symposium on Robot and Human Interactive Communication, 157-163, 2006.

[4] Arioui H., Kheddar A. and Mammar S., "A Model-based Controller for Interactive Force Reflecting Virtual Environment under Time Delay", Journal of Intelligent and Robotic Systems, 37(2), 193-207, 2003.

[5] Yanco H. A. and Drury J. L., "Classifying Human-Robot Interaction: An Updated Taxonomy", IEEE Conference on Systems, Man and Cybernetics, 2004.

[6] Colle E., Rybarczyk Y. and Hoppenot P., "ARPH: An assistant robot for disabled people", IEEE Conference on Systems, Man and Cybernetics, 2002.

[7] Fong, T. and Thorpe, C., "Vehicle teleoperation interface", Journal of Autonomous Robots, 11 (1), 9-18, 2001.

[8] S. Lee, G. S. Sukhatme, G. J. Kim and C. M. Park, "Haptic control of a mobile robot: A user study", In Proc. of IEEE/RSJ IROS, Lausanne, Switzerland, 2002.

[9] I. Farkhatdinov and Jee-Hwan Ryu, "Stability Analysis of Mobile Robot Teleoperation with Variable Force Feedback Gain”, EuroHaptics 2010, 177-182, 2010.

[10] I. Farkhatdinov, Jee-Hwan Ryu, Jinung An, “A Preliminary Experimental Study on Haptic Teleoperation of Mobile Robot with Variable Force Feedback Gain", IEEE Haptics Symposium 2010, 25-26 March, Waltham, Massachusetts, USA.

[11] O. Linda, Milos Manic, "Self-Organizing Fuzzy Haptic Teleoperation of Mobile Robot Using Sparse Sonar Data”, IEEE Trans. on Industrial Electronics, 2010.

[12] Shahdi and Sirouspour, "Model-based Decentralized Control of Time-delay Teleoperation Systems", Int. Journal of Robotics Research, 28, 376-394, 2009.

[13] Anderson R. and Spong M., "Bilateral Control of Teleoperators with Time Delay", IEEE Trans. on Automatic Control, 34 (5), 494-501, 1989.

[14] Niemeyer G. and Slotine J. J. "Towards force-Reflecting Teleoperation over the Internet", IEEE Int. Conference on Robotics and Automation, 1909-1915, 1998.

[15] Smith O. J. M., "A Controller to overcome dead time", ISA Journal, 1959.

[16] Adams R. J. and Hannaford B., "Stable Haptic Interaction with Virtual Environments", IEEE Trans. on Robotics and Automation, 15(3), 465-474, 1999.

[17] Arioui H., Temzi and L. Hoppenot Ph, "Force Feedback Stabilization for Remote Control of An Assistive Mobile Robot”, American Control Conference, 2011.

[18] Mana M., Arioui H., Kheddar A. and Mammar S., "Master model based time-delayed force feedback interaction: Experimental results", In Proc. IEEE Int. Symposium on Intelligent Control, 896-901, 2002. 